\title{
3-D CALCULATIONS OF FIELD DISTRIBUTION IN THE MEBT QUAD AND EFFECT OF THE FRINGE FIELD ON BEAM DYNAMICS *
}

\author{
A. Aleksandrov, SNS ORNL, Oak Ridge, TN 37830, USA \\ J. Staples, LBNL, Berkeley, CA 94720, USA
}

\begin{abstract}
Quadrupole magnets in the SNS Medium Energy Beam Transfer line (MEBT) are in close proximity to each other. The distance between iron cores of is comparable to the magnet aperture and therefore fringe fields of the magnets overlap. The magnetic field distribution of the SNS MEBT quadrupole magnets has been calculated using the 3-D MAFIA code. The effect of fringe field on beam dynamics is studied and corrections to quad settings are calculated.
\end{abstract}

\section{CALCULATION OF THE MAGNETIC FIELD DISTRIBUTION.}

The MEBT focusing system consists of 14 quadrupole magnets, 8 of $32 \mathrm{~mm}$ aperture and 6 of $42 \mathrm{~mm}$ aperture [1]. All quads have $45 \mathrm{~mm}$ long iron core. The distance between the edges of the iron cores of adjacent quads of the central triplet of $55 \mathrm{~mm}$ is comparable to their aperture of $42 \mathrm{~mm}$, therefore fringe magnetic fields of adjacent magnets can overlap affecting focusing properties of the triplet. In order to investigate the magnitude of the effect, 3D distribution of the magnetic field of the quads was calculated using the static solver from the MAFIA package.

\subsection{Magnetic field of a single quad.}

The calculated distributions of magnetic field gradient for $42 \mathrm{~mm}$ aperture quads with different excitation currents are shown in Fig.1. Effective gradients were calculated assuming effective length of $61 \mathrm{~mm}$ for $32 \mathrm{~mm}$ aperture quad and $66 \mathrm{~mm}$ for $42 \mathrm{~mm}$ aperture quad. Results are shown in the Table 1. Design values [1] are shown in parentheses for comparison. Note good agreement between calculated and design values. Difference is larger for larger currents, which can be explained by difference between design iron properties and material properties table used in MAFIA calculations. Dependence of quad focusing strength upon excitation current is shown in Fig.2.

\subsection{Effect of presence of the adjacent quad on the magnetic field distribution.}

In the present MEBT configuration three $42 \mathrm{~mm}$ aperture quadrupole magnets are placed in close proximity to each other. The presence of the iron core of the adjacent quad in the vicinity of the magnet gap can change distribution of the field. In order to
Table 1: Calculated effective gradient and deviation from the design value.

\begin{tabular}{|l|l|l|l|}
\hline$I[A]$ & $\varnothing[\mathrm{mm}]$ & $G[T / m]$ & $\delta G[\%]$ \\
\hline 166 & 42 & $11.492(11.70)$ & -1.8 \\
\hline 411 & 42 & $28.033(28.82)$ & -2.7 \\
\hline 500 & 42 & 33.627() & \\
\hline 100 & 32 & $12.075(11.996)$ & -0.9 \\
\hline 300 & 32 & $35.469(36.479)$ & -2.8 \\
\hline 500 & 32 & 51.559() & \\
\hline
\end{tabular}

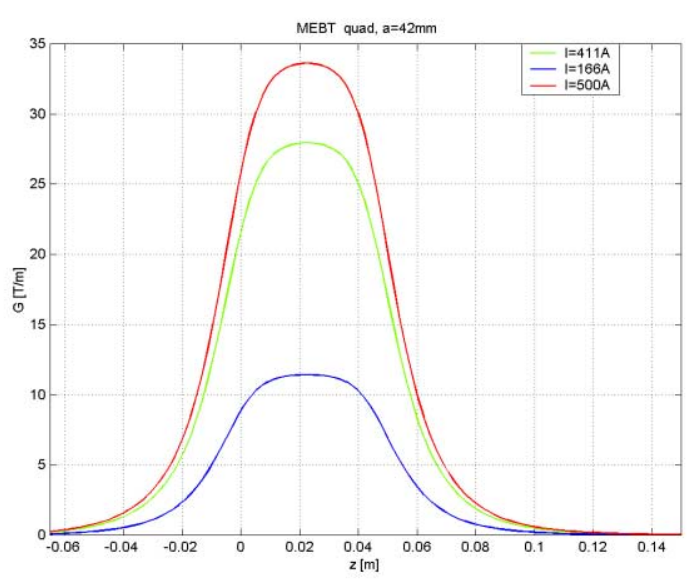

Figure 1: Distribution of the gradient in $42 \mathrm{~mm}$ quad

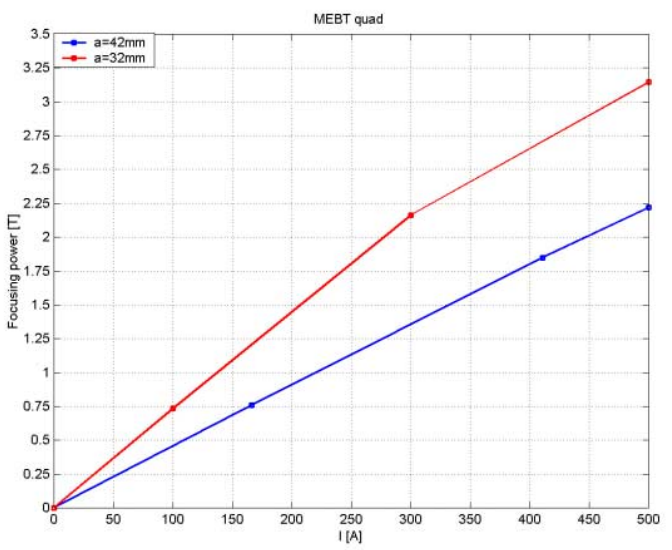

Figure 2: Dependence of integrated focusing strength upon current 
calculate the magnitude of the effect the second quad was added to the MAFIA model. Distribution of the magnetic field was calculated for different distances between quad centers with zero current in the coil of the second quad. Calculated gradient distributions are shown in Fig 3. Fig. 4 shows the difference between the perturbed field and field of the single quad. Calculated perturbation of the integrated focusing strength is presented in Table2.

Table 2. Relative change in focusing strength due to effect of adjacent iron core.

\begin{tabular}{|l|l|l|l|}
\hline$\Delta z[\mathrm{~mm}]$ & 60 & 80 & 100 \\
\hline$\delta G L[\%]$ & -7 & -2.38 & -0.7 \\
\hline
\end{tabular}

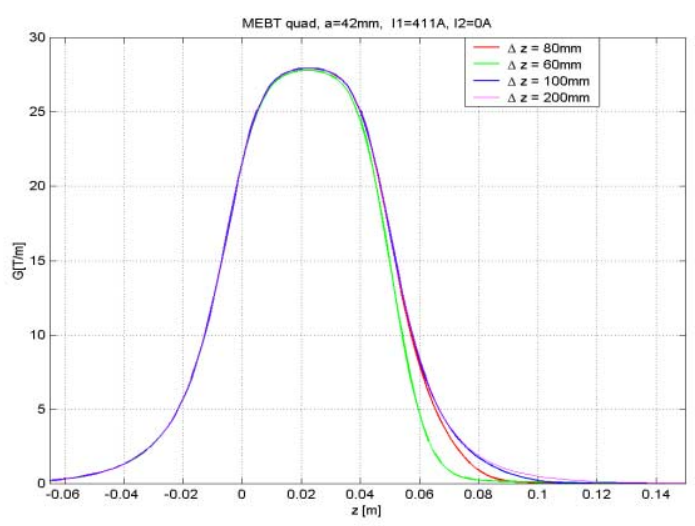

Figure 3: Distribution of gradient for different distances between adjacent quads

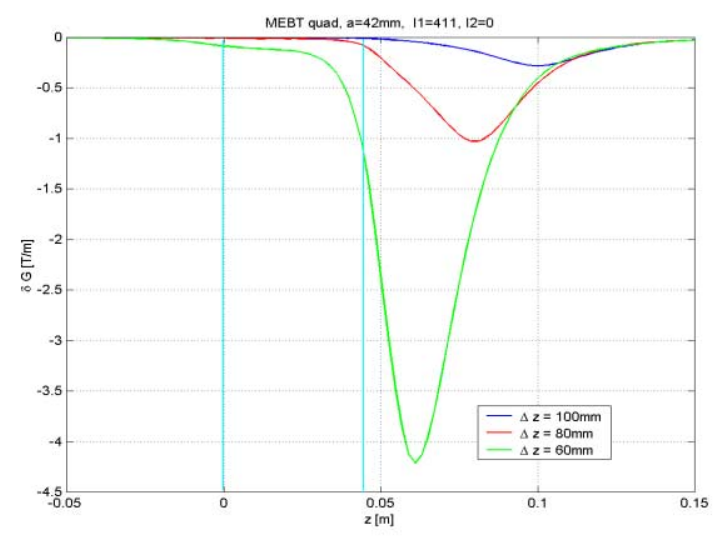

Figure 4. Perturbation of gradient for different distances between adgacent quads

\section{EFFECT OF FRINGE FIELD SUPERPOSITION ON THE FOCUSING PROPERTIES OF THE TRIPLET}

As can be seen from Fig.1. the magnetic field of 42 $\mathrm{mm}$ aperture quadrupole magnet stretches well beyond the physical edge of its core. When adjacent quads are placed close enough to each other their fringe fields overlap changing the focusing properties of the magnetic system. It was shown above that fringe field perturbation due to adjacent iron core is negligible for the actual separation of the MEBT quads. Therefore superposition of normalized gradient distributions of individual quad $g_{0}(z)$ shifted in accordance with quad position $z_{i}$ and weighted in accordance with design effective gradient $G_{i}$ can be used to represent combined gradient distribution of several adjacent quads:

$$
g(z)=\sum_{i=1 . .3} G_{i} \cdot g_{0}\left(z-z_{i}\right) .
$$

The calculated gradient distribution for one of a pair of triplet of quads used in the center section of the SNS MEBT is shown in Fig 5 together with the gradient distribution for individual non-interfering quads. One can see that overlapping of fringe fields of adjacent quads with opposite sign of the magnetic field leads to cancellation of the field in the overlap region thus reducing integrated effective focusing strength of each quad and shifting center of gravity of the distributions of individual quads.

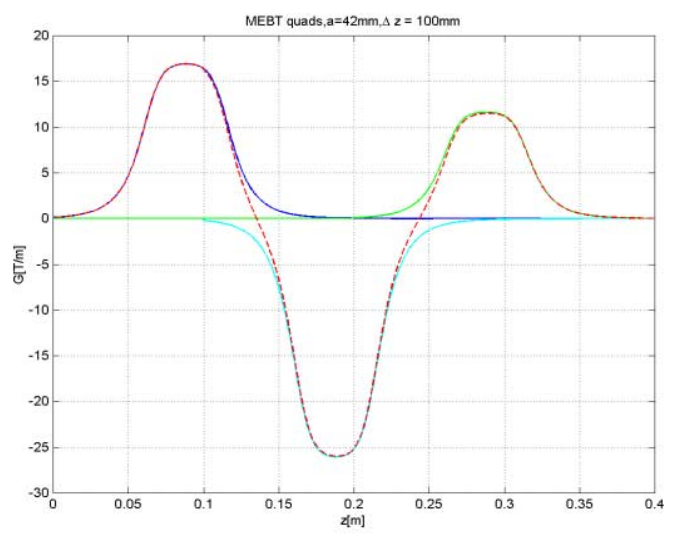

Figure 5. Gradient distribution in the triplet

The effect of fringe overlapping on the focusing properties was calculated in the following way. Transfer matrixes $M_{x}, M_{y}$ were calculated for actual gradient distribution $g_{0}(z)$ as $M=\prod_{j} d M_{j}$, where $d M_{j}$ is transfer matrix of thick hard edge quad 
of longitudinal length $d z$ and strength $g_{0}\left(z_{j}\right)$. Transfer matrixes of equivalent hard edge model $M_{0 x}, M_{0 y} \quad$ were calculated as $M=M_{d 1} \cdot M_{Q 1} \cdot M_{d 2} \cdot M_{Q 2} \cdot M_{d 3} \cdot M_{Q 3} \cdot M_{d 4}$, where $M_{d}$ is matrix of drift space, $M_{Q}$ is matrix of thick hard edge quad with gradient $Q$. Design values of $d_{2}, d_{3}, Q_{1}, Q_{2}, Q_{3}$ were taken from a PARMILA input file. Drift lengths $d_{1}, d_{4}$ were chosen to provide equal physical length of $M$ and $M_{0}$. Then effective gradients $G_{1}, G_{2}, G_{3}$ were adjusted to minimize relative difference $\delta M$ of transfer matrixes calculated as

$$
\delta M=\sqrt{\sum_{i, j=1,2} \frac{\left(m x_{i, j}-m x_{0 i, j}\right)^{2}}{m x_{0 i, j}^{2}}+\sum_{i, j=1,2} \frac{\left(m y_{i, j}-m y_{0 i, j}\right)^{2}}{m y_{0 i, j}^{2}}}
$$

The results are summarized in Table 3. The residual difference $\delta M$ is small but not negligible. To understand the magnitude of the effect, note that deviation of gradient of one quad by $.2 \%$ gives approximate matrix error $\delta M=2.5 \%$. For further decreasing of the difference between real triplet and hard edge quads model, asymmetry of field distribution can be taken into account by shifting centers of hard edge model quads. The result is shown in Table 4. The shifts of ideal quad centers from the centers of the real quad cores by $-5 \mathrm{~mm}$ for the first quad in the triplet and $+6 \mathrm{~mm}$ for the third quad and subsequent adjustment of effective gradients reduces the error to a negligible value of $.3 \%$.

\section{CONCLUSION}

Analysis of the magnetic field distribution of the MEBT quads using 3D calculation shows that the presence of the iron core of the adjacent quad close to the magnetic gap doesn't significantly affect gradient, while overlap of fringe fields of the quads in the triplet change the transfer matrix of the focusing system considerably. Adjusting quad strengths and slightly changing positions of hard edge quads in the model can correct the effect. Correction coefficients are calculated. The required correction to quad strength of about $10 \%$ is significant and has to be taken into account for initial quad setting during commissioning. Effect of quads proximity on higher multipoles of the field distribution was investigated as well but it is negligible and not discussed here.

Table 3. Calculated gradients minimizing deviation of real transfer matrix from hard edge model.

\begin{tabular}{|l|l|l|l|l|}
\hline & $G_{1}[T / m]$ & $G_{2}[T / m]$ & $G_{3}[T / m]$ & $\delta M$ \\
\hline $\begin{array}{l}\text { Calculated gradient } \\
\text { (design value) }\end{array}$ & -18.1442 & 28.3765 & -12.7880 & $2.36 \%$ \\
\hline Gradient deviation & $(-17.0)$ & $(26.2)$ & $(-11.7)$ & \\
\hline
\end{tabular}

Table 4. Calculated gradients minimizing deviation of real transfer matrix from hard edge model with shifted model quads. $\delta z 1=-5 m m, \quad \delta z 2=+6 m m$

\begin{tabular}{|l|l|l|l|l|}
\hline & $G_{1}[T / m]$ & $G_{2}[T / m]$ & $G_{3}[T / m]$ & $\delta M$ \\
\hline Calculated gradient & -18.8303 & 29.5482 & -13.2605 & $0.33 \%$ \\
(design value) & $(-17.0)$ & $(26.2)$ & $(-11.7)$ & \\
\hline Deviation & $+10.61 \%$ & $+12.78 \%$ & $+13.34 \%$ & \\
\hline
\end{tabular}

\section{REFERENCES}

[1] John Staples, private communication

*Work performed under auspices of the US Department of Energy, contract no. DE-AC05-00OR22725 with UTBatelle, LLC for ORNL. 